\title{
Study on Electroscope of UHV Equipment based on ZnS Material Kang Chen ${ }^{1}{ }^{\star}$, Bin Song ${ }^{1}$, En-wen $\mathrm{Li}^{1}$, Li-nong Wang ${ }^{1}$ and Zheng Cheng ${ }^{2}$ \\ ${ }^{1}$ Wuchang District, Wuhan City, Hubei Province of China \\ ${ }^{2}$ Electric Power Control Center of Jiangxi Province,China
}

\section{Keywords: ZnS, High Voltage Equipment, Electroscope}

\begin{abstract}
UHV equipment charged instructions plays a very important role for the maintenance of workers' personal safety. Low-voltage side usually use the electroscope, but there is no appropriate electroscope for the high-voltage side. As the voltage increases, the greater the safety distance is required, the length and deflection of inspection pole cannot meet the requirements. In this paper, $\mathrm{ZnS}$ electro chromic devices were fabricated, and the devices were encapsulated by screen printing. We obtained the volt-ampere characteristics through the power-on test, and the light-emitting situation, and the devices were tested under the actual high-voltage electric field. Experiments show that the device has the effect of inspection, which can be installed directly on the surface of charging equipment. So it can be used for charging indicator.
\end{abstract}

\section{Introduction}

At present, the contact high-voltage type electroscope has been widely used in the maintenance of high voltage power transmission and transformation equipment. However, the overall quality is large, the operation is not convenient. To solve this problem, we developed a $\mathrm{ZnS}$ device for high voltage power transmission equipment. It can be painted in the corresponding location of the UHV power transmission and transformation equipment, which is to characterize whether the equipment is charged. it has good qualities with high reliability, easy operation, low cost, and can be used in outdoor long-term.

\section{Production of Material}

A thin film electroluminescent device was prepared on the basis of $\mathrm{ZnS}$-doped electroluminescent phosphor powder prepared by high-temperature solid-state method. The specific technical process of each process is as follows:

a) Pretreatment: Cut a certain size of the ITO film; put it into the oven 120 degrees Celsius, insulation 30min.

b) Ingredients:mix the phosphor powder with 9802A glue in a certain proportion, and then use the stirring rod. Weigh and add a certain amount of cyclohexanone solvent, until the viscosity is appropriate, and fully stir well.5g BaTiO3 slurry was weighed (the mixture of dielectric material BaTiO3 and the corresponding dielectric gel) with the container, and stir well.

c) Screen printing: clean the screen with cyclohexanone solvent carefully and dry with a hair dryer, then fix the ITO sheet on the screen printer. Then, mix the deployment of a good phosphor slurry fully with a scraper printing, back and forth twice. The printed ITO film of the phosphor was removed and placed in a 100 degree oven for 30 minutes. Then the insulating paste BaTiO3 (100 degrees, insulation 30min), conductive silver paste (120 degrees Celsius, insulation $45 \mathrm{~min}$ ) is with the same method of printing.

d) Package: There are two ways to seal the device. One method is to coat a thicker $\mathrm{SiO}_{2}$ film directly after all the layers have been prepared. The other is to fill the conductive film with silicone oil. We choose the second one.

Electroluminescent devices are made of thin sheets. The outer lead of the device is composed of transparent conductive film (ITO) and silver film. And we use the metal pin for connection. 


\section{Test of Materials}

The sheets emit bright blue-green light when the $\mathrm{ZnS}$ sheet is passed through the alternating current.

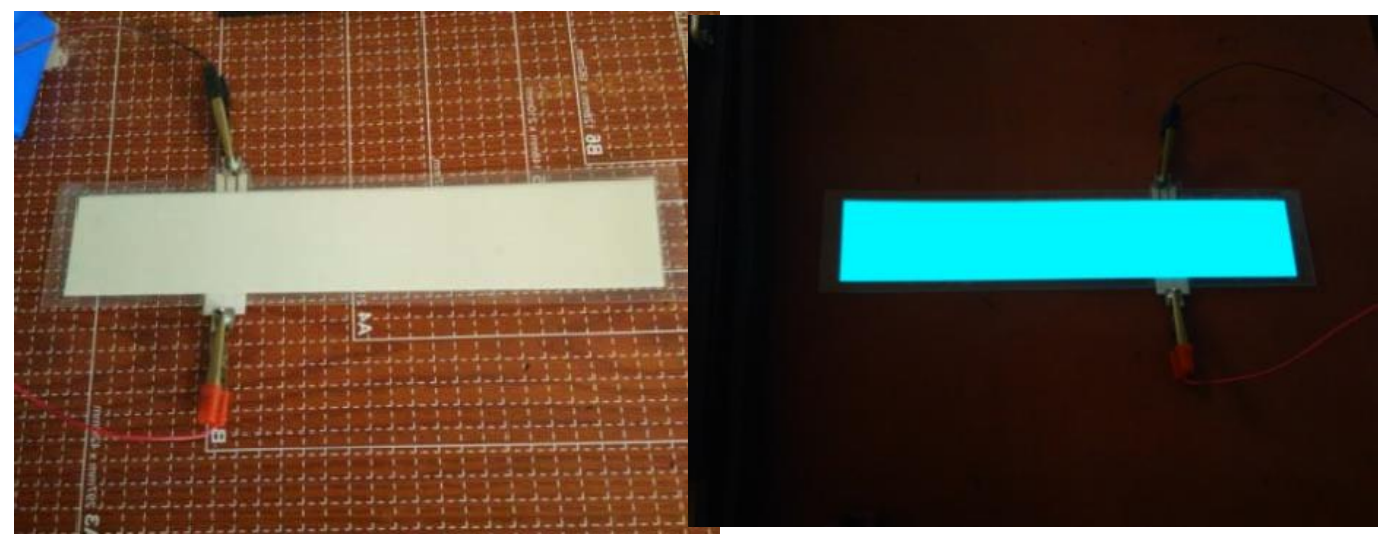

Figure1. ZnS device test

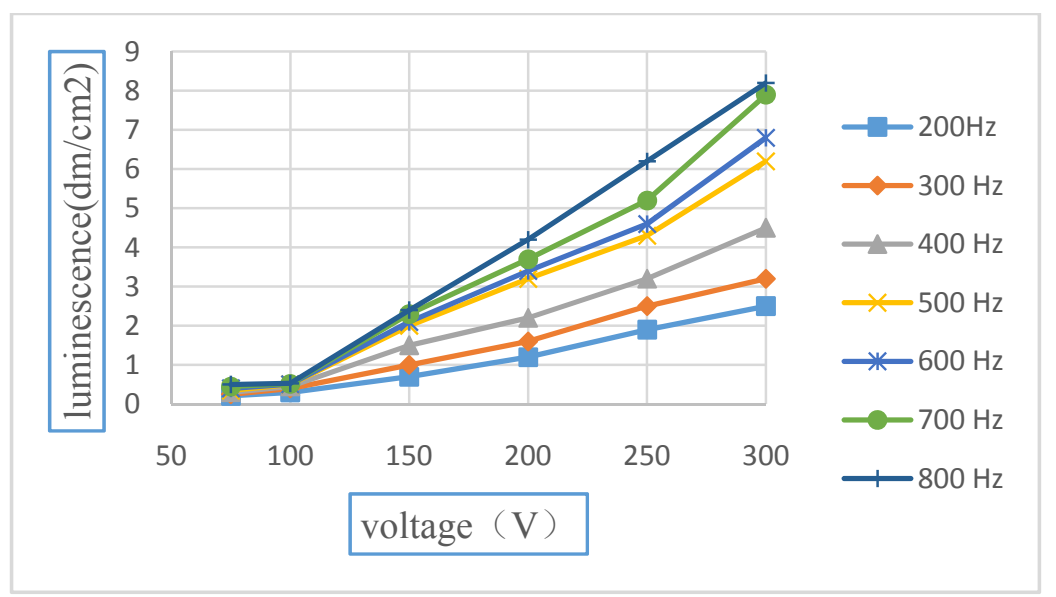

Figure2. Luminous characteristic curve of $\mathrm{ZnS}$ device

$\mathrm{ZnS}$ was directly placed in an electric field for experiments. When an alternating current voltage is applied between the two electrodes of the $\mathrm{ZnS}$ color changing device, a strong electric field is established inside the material. Electrons in the trending materials accelerate electrons to strike the luminescent center, causing excitation or ionization of the luminescent centers, and then directly emit luminescence centers after the collision, or recombine luminescence by the ionized electrons.

The surface of High-voltage power transmission equipment, which is charged will have a strong electric field. The electrons in the material accelerate to strike the luminescent center under the action of electric field, causing the excitation center of excitation or ionization. The luminescence center will emits light after the collision excitation, the ionized electrons which are trapped will also recombine to emit light.

Under the action of the strong electric field, the electron inside the material can be accelerated to hit the light-emitting center and cause to emit light. So the $\mathrm{ZnS}$ device will be placed directly into the high-voltage electric field for testing.

We use $100 \mathrm{kV}$ transformer step upvoltage to generate electric field, simulating the strong electric field of high-voltage power transmission equipment. The experimental transformer as shown in the figure below. 


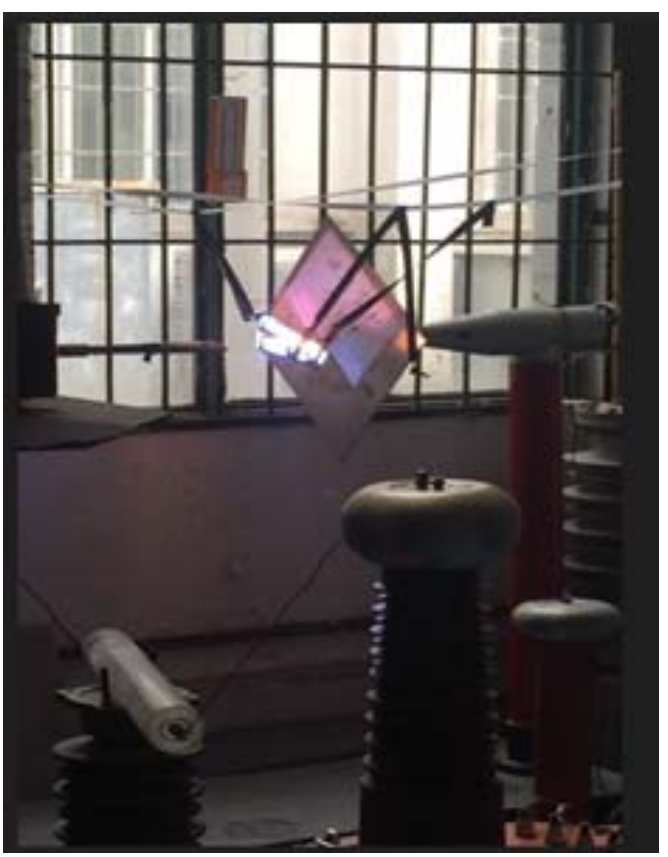

Figure3.High voltage test chart

Under the action of the electric field, the $\mathrm{ZnS}$ material give out light when the voltage reaches $40 \mathrm{KV}$.

After measurement, the flow of the current table is as follows:

Table 1. Current flowing through $\mathrm{ZnS}$ under the action of an electric field

\begin{tabular}{|l|l|}
\hline Applied voltage $(\mathrm{kV})$ & current $(\mathrm{mA})$ \\
\hline 4 & - \\
\hline 8 & - \\
\hline 12 & $0.007 \sim 0.015$ \\
\hline 16 & 0.036 \\
\hline 20 & 0.055 \\
\hline 24 & 0.072 \\
\hline 28 & 0.087 \\
\hline 32 & 0.1 \\
\hline 36 & 0.115 \\
\hline 40 & 0.127 \\
\hline 44 & 0.144 \\
\hline 48 & 0.156 \\
\hline 52 & 0.17 \\
\hline 56 & 0.189 \\
\hline 60 & 0.203 \\
\hline
\end{tabular}

\section{Conclusions}

The test results show that the $\mathrm{ZnS}$ device can change color in the electric field and indicate the charged status of the high-voltage power transmission and transformation equipment. In order to make the $\mathrm{ZnS}$ device emit light, it is necessary to form a strong electric field between the thin film layers, by adding a certain voltage between the upper and lower membrane layer. And the microampere current is needed to be injected into the material. A strong electric field and a certain amount of electrons are necessary for the material to emit light. This material can be used in the inspection of high voltage power transmission equipment instructions with features of briefness and convenience. 


\section{References:}

[1] Tang Chunjiu, Jiang Xueyin. Dynamic ZnS: Mn / SrS: Ce / ZnS: Mn Thin Film Electrodical Study [J]. CHINESE JOURNAL OF LUMINESCENCE, 1996, 17(4):317-321.

[2] Li Xiaoli. Electrodynamics Material-like-use [J]. Science Resources, 2011(2):3-3.

[3] Liu Ping. New Organic Electrodeposition Material Compensation Material Control and Performance [J]. Materials research, 2010, 04(4):321-324.

[4] Yuan Xiao. Research on electrochromic materials and exhibition exhibition [J]. Advanced Materials Industry, 2014(5):14-18.

[5] Zhang Zhenglin. For electrodepositing materials and for use [J]. Electronic source material, 1999(1):32-36.

[6] ChenYongjun, Lin Yongchang. Electrochromic device [J]. Optoelectronic Technology, 1996(1):42-46.

[7] Weng Duo. Research and Development of Dark Electrochromic Devices Based on Spectral Complementary Absorption Principle [D]. University of Science and Technology of China, 2016.

[8] Li Mengke, Xu Ningning, Yang Yongchao. Preparation and Characterization of MWNTs / PTH Electrochromic Devices [J]. Journal of Liaoning Normal University, 2016, 39(2):186-191.

[9] Wei Youxiu, Chen Mu, Liu Weiming. Research Progress and Application of Electrochromism Technology [J]. Journal of Aeronautical Materials, 2016, 36(3):108-123.

[10] Zhou Ding. Controllable preparation and performance improvement of tungsten oxide based electrochromic materials [D]. Zhejiang University, 2016. 